\title{
Random and channeled energy loss of 33.2-TeV Pb nuclei in silicon single crystals
}

\author{
S. Pape Møller, ${ }^{1 *}$ V. Biryukov, ${ }^{2}$ S. Datz, ${ }^{3}$ P. Grafström, ${ }^{4}$ H. Knudsen, ${ }^{5}$ H. F. Krause, ${ }^{3}$ C. Scheidenberger, ${ }^{6}$ U. I. Uggerhøj, \\ and C. R. Vane ${ }^{3}$ \\ ${ }^{1}$ Institute for Storage Ring Facilities, Aarhus University, DK-8000 Aarhus C, Denmark \\ ${ }^{2}$ Institute for High Energy Physics, Beam Department, 142284 Protvino, Moscow Region, Russia \\ ${ }^{3}$ Oak Ridge National Laboratory, P.O. Box 2008, Oak Ridge, Tennessee 37831-6377 \\ ${ }^{4}$ CERN, CH-1211 Geneve 23, Switzerland \\ ${ }^{5}$ Institute of Physics and Astronomy, Aarhus University, DK-8000 Aarhus C, Denmark \\ ${ }^{6}$ Gesellschaft für Schwerionenforschung mbH, Planckstrasse 1, D-64291 Darmstadt, Germany
}

(Received 10 April 2001; published 15 August 2001)

\begin{abstract}
Measurements of the energy loss and the energy-loss distributions of $160 \mathrm{GeV} / \mathrm{amu}$ fully stripped lead ions traversing a silicon single crystal are presented. The energy loss is measured using the silicon crystal as an intrinsic detector. Hence the measured energy loss is a restricted energy loss excluding very large energy transfers. For random incidence, the observed energy-loss distributions are very narrow and Gaussian-like. For well-channeled particles, the energy loss is strongly reduced as compared to so-called random particles. The observed energy loss is compared to calculations as well as simulations. Due to the small straggling, the energy-loss distributions are reflecting directly the distribution in transverse energy.
\end{abstract}

DOI: 10.1103/PhysRevA.64.032902

PACS number(s): $34.50 . \mathrm{Bw}, 61.85 .+\mathrm{p}$

\section{INTRODUCTION}

The energy loss per unit distance, the stopping power, and the distribution of energy losses, the straggling are key parameters in the understanding of the passage of charged particles through matter. Since the first investigations at the beginning of the last century, each time a new energy or particle regime has been entered, new phenomena have appeared. Hence the description and the calculation of energy loss have evolved over time using models including new important processes. From a more practical point of view, an understanding of slowing-down phenomena is also necessary. Two prime examples, relying on an accurate description of the passage of charged particles through matter, are radiology and detector development.

Since the discovery and first treatment of the channeling effect [1], stopping phenomena for channeled particles have been studied. There is an interplay between the channeling effect and the slowing-down process. The channeling effect has been used to vary the impact-parameter distribution and hence to study the impact-parameter dependence of the stopping power. Conversely, measurements of stopping powers for channeled particles have been used to obtain information about the trajectories of the channeled particles. As a reference to the energy loss of relativistic random and channeled particles, we refer to the first general survey for relativistic particles [2], and to the more recent paper [3] on the energy loss of channeled particles in bent crystals.

Recently, fully stripped ions at ultrarelativistic energies have become available at CERN. This led to the discovery [4] and subsequent explanation [5] of the now called nuclearsize effect, or Lindhard-Sørensen effect, which leads to a reduction of the stopping power as compared to that found from the Bethe-Bloch formalism, for large values of the Lor-

\footnotetext{
*Corresponding author. Email address: FYSSP@IFA.AU.DK
}

entz factor. The reduction appears for close collisions when the de Broglie wavelength of the projectile becomes smaller than the nuclear dimension. Clearly, in this case the collisions can no longer be considered as collisions between pointlike particles.

In the present investigations, we use intrinsic silicon crystals for detection of the energy loss. In this way it is the deposited energy, which is measured, and energetic electrons may escape the target. Hence, this so-called restricted energy loss excludes high-energy transfers from close collisions, and the above-mentioned nuclear-size effect has no influence as it appears for close collisions only. Instead the aim of the present experiment was to obtain a general survey of the energy-loss process for channeled and random incidence for highly charged relativistic lead ions, and in particular to try to take advantage of the small straggling as described in the following.

\section{EXPERIMENT}

The experiment was performed in the $H 2$ beamline in the north area of the SPS at CERN. A beam of $160 \mathrm{GeV} / \mathrm{amu}$ of ${ }^{206} \mathrm{~Pb}^{82+}$ ions with an intensity of around $1000 / \mathrm{sec}$ during the spill was incident on a 0.3 -mm-thick silicon crystal mounted in a precision goniometer. A system of scintillators in coincidence or anticoincidence defined the usable fraction of the beam hitting the active area of the crystal. The root-meansquare divergence of the beam was around $50 \mu \mathrm{rad}$. The goniometer had a minimum step angle of $1 / 10000^{\circ}$ $=1.7 \mu \mathrm{rad}$. The crystal was aligned observing the lowenergy loss for planar or axially channeled ions. In this way it was possible to align the beam direction with planar or axial directions to a precision much better than the beam divergence.

Since there was material, mainly vacuum windows, in the beamline, the beam did not consist of lead nuclei only, but instead of a mixture of fragments produced by nuclear colli- 


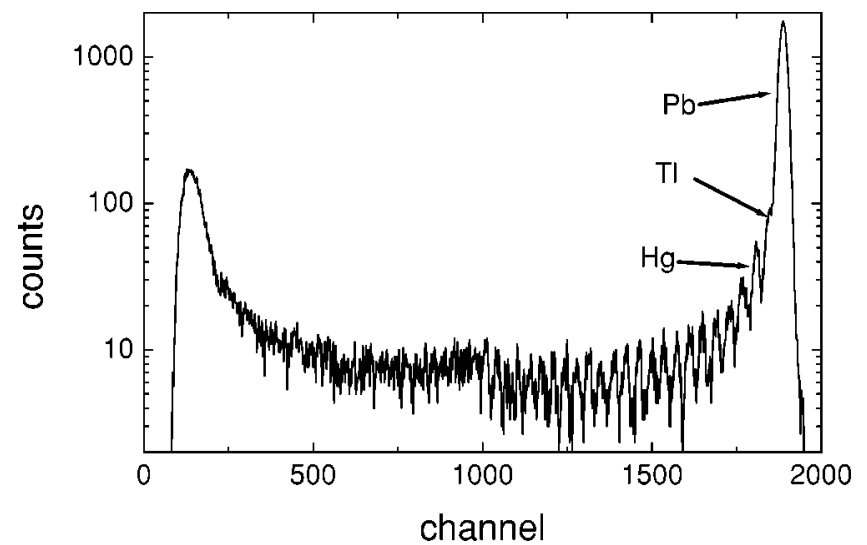

FIG. 1. Distribution of signals from the MUSIC detector showing the distribution of charges in the beam. Note the logarithmic vertical scale.

sions in the material. The nuclear charge of the incident particles were tagged by using the energy deposition in a gasfilled ionization chamber, a so-called MUSIC detector [6]. A spectrum from this detector is shown in Fig. 1, and the large ${ }^{208} \mathrm{~Pb}^{82+}$ peak and the much smaller peaks from nuclei of lower atomic numbers are clearly visible. The peaks corresponding to different atomic numbers cannot be resolved completely, since only one anode of the MUSIC detector [6] was used.

Thin electrodes on the surfaces of the crystal were used to make it into a surface-barrier detector, which could register the deposited energy. An amplifier system consisting of a pre- and a main-amplifier created a signal appropriate for the data-taking system analog-to-digital converter. The very high charge of the projectiles results in very large ionization in the silicon crystal, and hence care was taken to avoid saturation effects. As seen above, there are nuclei of practically any atomic number in the beam. This means that an online check of saturation effects could be made by observation of the silicon-detector signal relative to the MUSIC signal. In Fig. 2 is shown a two-dimensional plot of these two signals, and the

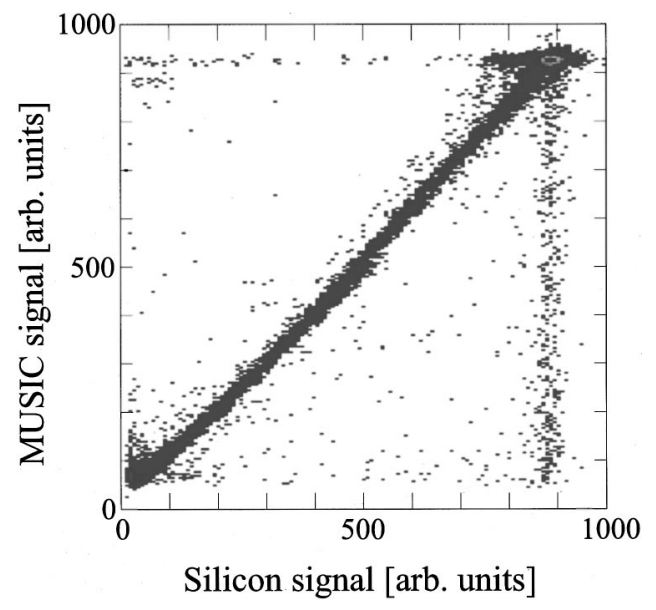

FIG. 2. Two-dimensional distribution of signals from the silicon detector versus the MUSIC detector showing the linearity of both detectors. approximate linearity is apparent.

The measured energy loss can be calibrated on an absolute scale by the use of radioactive sources, if the absolute thickness is known and if the detector is fully depleted. Unfortunately this was not possible in the present experiments, since the detector could not be fully depleted owing to electrical breakdown. Hence the energy-loss measurements presented here are only relative, and the thickness of the active layer is only known to be significantly less than the physical thickness of $0.3 \mathrm{~mm}$.

\section{THEORETICAL CONSIDERATIONS AND EXPECTATIONS}

The energy loss of relativistic particles traversing a target of thickness $\Delta x$ is described by the Bethe formula [7]

$$
\Delta E=\Delta x \frac{4 \pi e^{4} N Z_{2}}{m v^{2}} Z_{1}^{2}\left[\ln \left(\frac{2 m v^{2} \gamma^{2}}{I}\right)-\beta^{2}-\delta / 2+\Delta L\right],
$$

where $Z_{1}$ and $Z_{2}$ are the projectile and target atomic numbers, $N$ the target atomic density, $I$ the mean ionization potential, $v$ the projectile velocity, $\beta=v / c$ and $\gamma=(1$ $\left.-\beta^{2}\right)^{-1 / 2}$ the Lorentz factor. The last two correction terms are the Fermi-density effect $\delta$ and the Lindhard-Sørensen correction $\Delta L[5]$. For highly charged ions, the feature of paramount importance is the $Z_{1}^{2}$ front factor in the Bethe formula resulting in an energy loss, which is around four orders of magnitude higher for lead nuclei than for protons.

The restricted energy loss, excluding energy transfers larger than $E_{0}$, is given approximately by [7]

$$
\begin{aligned}
\Delta E_{\text {restr. }}= & \Delta x \frac{2 \pi e^{4} N Z_{2}}{m v^{2}} Z_{1}^{2}\left[\ln \left(\frac{2 m v^{2} \gamma^{2} E_{0}}{I^{2}}\right)\right. \\
& \left.-\frac{E_{0}}{2 m c^{2} \gamma^{2}}-\beta^{2}-\delta\right] .
\end{aligned}
$$

The restriction energy $E_{0}$ corresponds to the energy of an electron having a range of half the target thickness. The Fermi-density effect saturates the logarithmic increase of the restricted energy loss at large values of $\gamma$ at the so-called Fermi plateau.

For channeled particles, a path-dependent average energy loss for relativistic particles was calculated by Esbensen and Golovchenko [8]

$$
\begin{aligned}
\Delta E(\mathbf{b})= & \Delta x \frac{2 \pi e^{4} N}{m v^{2}} Z_{1}^{2}\left[\left[Z_{2}+Z_{2}(b)\right]\left\{\ln \left(\frac{2 m v^{2} \gamma^{2}}{I}\right)-\beta^{2}\right\}\right. \\
& \left.-Z_{2} \delta+C(\mathbf{b})\right]
\end{aligned}
$$

where $N Z_{2}(\mathbf{b})$ is the local electron density at position $\mathbf{b}$ in the transverse plane and where $C(\mathbf{b})$ is a velocityindependent term dependent on the local electron density at position $\mathbf{b}$. The same authors also calculated the reduction in the well-defined leading edge of the energy-loss distribution for channeled particles $[8,2]$.

When the energy loss of the particles is sufficiently large, 


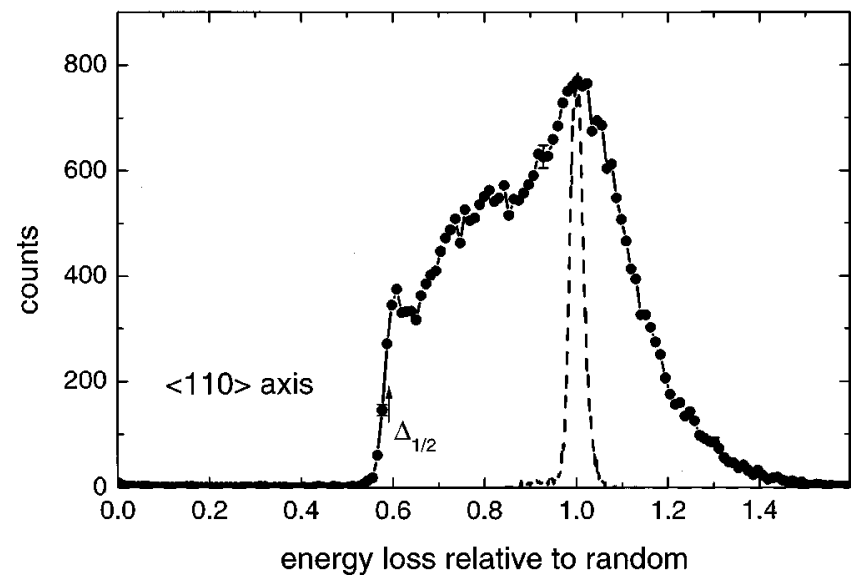

FIG. 3. Distribution of energy losses for beam incidence along the $\langle 110\rangle$ axial direction (filled circles). The distribution for a nonaligned beam is shown by the dashed curve. The leading-edge energy loss from Refs. [2,8] is marked by the arrow.

that the number of collisions of all types is much larger than one, the energy-loss distribution is Gaussian, and given by

$$
f(x, \Delta)=\frac{1}{\sqrt{2 \pi} \Omega} \exp \left(-(\Delta-\bar{\Delta})^{2} / 2 \Omega^{2}\right) .
$$

Here $x$ is the target thickness, $\Delta$ the energy loss, and $\bar{\Delta}$ the average energy loss. The standard deviation is given by

$$
\Omega^{2}=\Delta x \int_{E_{\min }}^{E_{\max }} d \sigma(E) E^{2}
$$

where $E_{\min }$ and $E_{\max }$ are the minimum and maximum energy transfers, respectively. The Rutherford cross section is a good approximation to the differential cross section $d \sigma(E)$ in the present case as the integral is heavily weighted towards large energy transfers. In the nonrelativistic case, the well-known Bohr expression for the straggling is obtained. Only the upper limit in the integral is important, and it corresponds in the present case to $E_{0}$ and not the very much larger maximum energy transfer in a collision between a heavy projectile and an electron. Hence the width of this distribution of deposited energies is much smaller than the corresponding width of the distribution of energy losses proper.

\section{RESULTS AND DISCUSSION}

The deposited energy-loss distribution for random incidence is shown in Fig. 3 as the dashed curve. In the remainder of the present article, we will be referring to restricted or deposited energy losses unless otherwise mentioned. The energy-loss distribution is seen to be a Gaussian-like distribution. The width is determined solely by the detector resolution amounting to approximately $20 \mathrm{keV}$ (full width at half-maximum) since the intrinsic width calculated according to the above formula is much smaller. The very small tail towards lower energies is not believed to have any physical significance, and is most probably stemming from particles

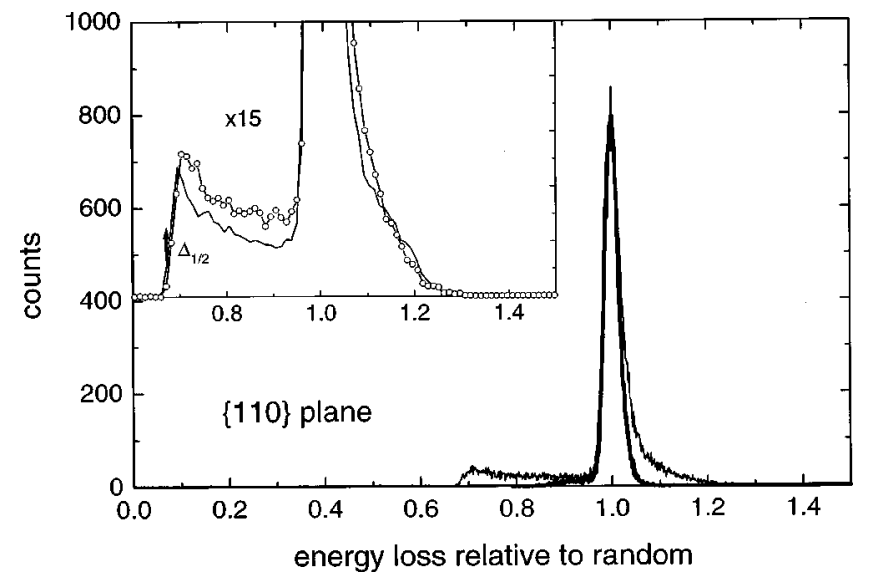

FIG. 4. Energy-loss distribution for beam incidence along the $\{110\}$ plane (thin-solid line). The distribution for a nonaligned, random incidence, beam is shown by the thick-solid curve. The inset shows the data for aligned incidence represented by open circles. The leading-edge energy loss from Refs. [2,8] is marked by the arrow. The simulated energy-loss distribution is shown by the fulldrawn line in the inset.

hitting the edge of the detector due to a finite inefficiency in the veto scintillator in front of the detector.

When the crystal is aligned with the $\langle 110\rangle$ axial direction, a much broader distribution is found as can be seen in Fig. 3 as filled circles. Here it should be realized that the divergence of the beam, around $50 \mu \mathrm{rad} \mathrm{rms}$, is much larger that the critical channeling angles. For the $\langle 110\rangle$ axis the calculated Lindhard angle is $\psi_{1}=22 \mu \mathrm{rad}$ and for the $\{110\}$ planes the planar channeling angle is $\psi_{p}=8 \mu \mathrm{rad}$. This means that the distribution includes energy losses from particles that are axially channeled, planar channeled and particles traversing the crystal in the transition region, the so-called strings of strings region. The distribution is seen to include particles giving an energy loss of up to $45 \%$ above the random value. Such large energy losses occur for planar-channeled particles with large transverse energies. A well-defined quantity is the energy loss of the best-channeled particles, which are axially channeled particles with small transverse energies. This quantity is represented by the low-energy edge of the distribution and amounts to $58 \%$ of the random energy loss. It is compared to the calculated leading-edge energy loss from the Esbensen and Golovchenko formalism [8] marked with an arrow in Fig. 3. Agreement to the few percent level is seen.

For planar-channeled particles the spectra for incidence along the $\{110\}$ and $\{111\}$ planes are shown in Figs. 4 and 5 as thin-solid lines. The distribution for random incidence is also shown for comparison as a thick-solid line. The random spectrum is scaled to have the same height as the spectrum for aligned incidence. Again it should be pointed out, that the divergence of the beam is much wider than the planarchanneling angle, which means that there is a large number of nonchanneled particles, as is also apparent from the large peak of random energy losses. A magnified view of the spectra for aligned incidence is shown in the upper left-hand side insets. The experimental data are here shown as connected open circles. A large energy-loss tail is seen in both spectra extending up to $30 \%$ and $40 \%$ above the random value for 


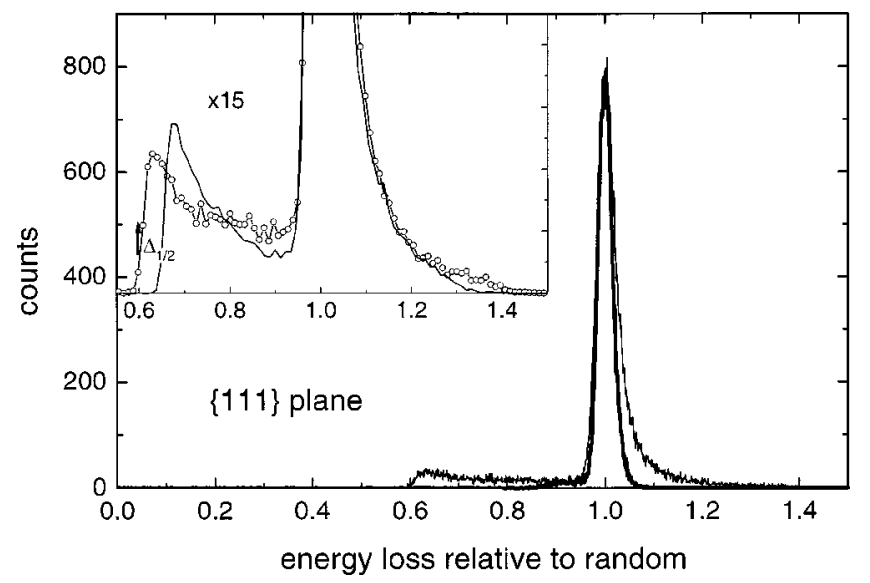

FIG. 5. As Fig. 4, but for the $\{111\}$ plane.

the $\{110\}$ and $\{111\}$ planes respectively, corresponding to particles with large transverse energies. The planar electron density is higher for the $\{111\}$ planes as compared to the $\{110\}$ planes, in accordance with the higher energy losses observed for the $\{111\}$ plane. Due to the divergent beam there will be a wide transverse energy distribution including particles with transverse energies from zero up to large transverse energies corresponding to angles much larger than the critical angle. This is reflected in the energy-loss distribution, which exhibits energy losses from above the random value down to the energy loss of the best-channeled particles. The leading edge of the distribution corresponding to the energy loss of the best-channeled particles is seen to be at $61 \%$ and $69 \%$ for the $\{111\}$ and $\{110\}$ planes, respectively. These values are compared to the Esbensen and Golovchenko values [8] and again agreement to the few percent level is observed. Another noteworthy feature on the experimental spectra is that the energy-loss distribution for the channeled particles peaks at the lowest energy loss corresponding to the best-channeled particles. This is a result of the transverse energy distribution. Although the present beam has a divergence larger than the critical channeling angle, the distribution in transverse energies peaks at low energies due to the almost harmonic form of the planar transverse potential. In order to make a more quantitative comparison, restricted energy losses in the silicon crystals were simulated using the Monte Carlo code CATCH [9], which employs the Esbensen and Golovchenko formula for path-dependent energy losses [8]. Fully stripped $\mathrm{Pb}$ ions were tracked through the crystal lattice with a step size of $0.5 \mu \mathrm{m}$. The electron density and interplanar field used in the simulation was calculated from a thermallyaveraged Molière potential. In every step, a number of electronic scattering events were generated according to a $1 / E^{2}$ distribution for the energy transfer $E$. If an electron was carrying an energy $E$ greater than a specified cut-off value, this energy transfer was not counted in the "restricted" energy loss of the considered particle (as the corresponding electron most likely escaped the depletion zone thus carrying away the energy). One poorly known input parameter to the simulation is required, namely, the cut-off restriction energy. This was taken to be $30 \mathrm{keV}$ corresponding to a thickness of the depleted layer of a few tens of microns.

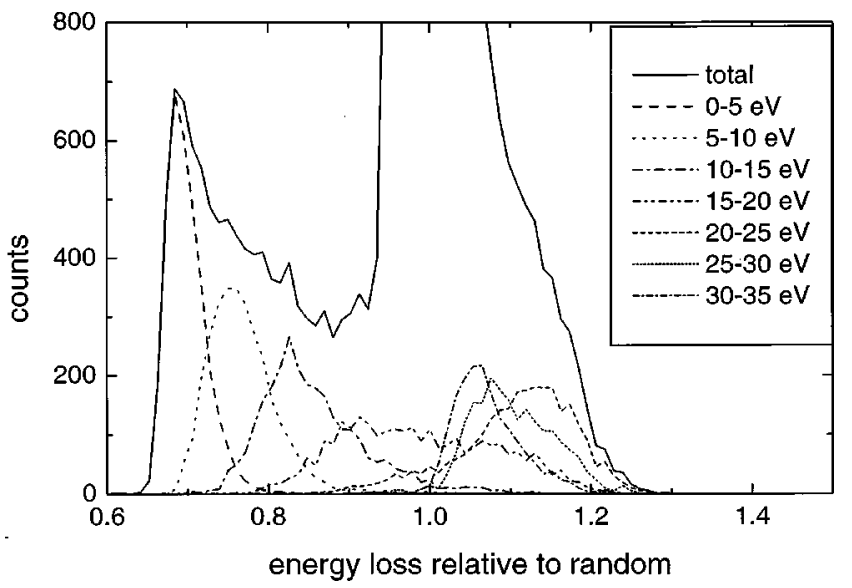

FIG. 6. Energy-loss distribution for the beam incident along the $\{110\}$ plane according to the CATCH simulation (full-drawn curve). The partial contributions from seven transverse energy intervals are also shown.

The simulated energy-loss distributions shown in the inserts of Figs. 4 and 5 as solid lines are normalized so that the area of the simulated and the experimental distributions are equal. We observe that the simulated distribution reproduces qualitatively the shape of the experimental distribution, although the exact form is not reproduced. We also observe from Figs. 4 and 5, that the measured energy loss of the best-channeled particles cannot be reproduced in the simulation for both planes. This is partly explained by the use of the Molière potential in the simulation, which apparently is not reproducing the electron density very well at large distances from the atomic nuclei. A better agreement with reference [8] for the leading-edge energy loss is due to the use of the Doyle-Turner potential in this calculation. Furthermore the position of the leading edge relative to the random energy loss depends somewhat on the choice of the restriction energy.

The simulated distributions in Figs. 4 and 5 consist of contributions from particles of different transverse energies obtained during surface transmission. The partial contributions to the energy-loss distribution from seven transverse energy intervals are shown in Fig. 6 for the $\{110\}$ plane. We observe as expected, that the particles with the lowest transverse energies give the smallest energy loss. In addition, the highest energy loss is obtained for particles with transverse energies in the interval $20-25 \mathrm{eV}$, i.e., around the height of the transverse potential. It is well known from channeling theory [1], that it is these particles, which gives an energy loss higher than random. For very large transverse energies the energy loss approaches the random value. This figure supports the above interpretation of the measured energyloss distributions; in particular that the low-energy edge is determined by the best-channeled particles and that particles with high transverse energies around the critical energy give an energy loss larger than random.

\section{CONCLUSIONS AND OUTLOOK}

The energy loss and the energy-loss distribution of channeled and random highly charged relativistic particles have 
been measured. For random incidence a very narrow, Gaussian distribution is seen. This is in contrast to incidence of protons, where a Landau distribution is observed. This difference is caused by the high charge of the projectiles. It was found that the Esbensen and Golovchenko theoretical minimum energy loss for channeled particles agrees well with our measurements. Further, the "channeled" energy-loss distributions could be explained on the basis of the distribution of the particles in transverse energy. A simulation qualitatively confirms this interpretation.

Even better quantitative investigations of the energy loss of channeled particles could be obtained using a beam with a smaller divergence, and using a fully depleted detector. Also a simulation using a better potential and electrondensity distribution like the Doyle-Turner potential would be interesting.
[1] J. Lindhard, Mat. Fys. Medd. K. Dan. Vidensk. Selsk. 34, 14 (1965).

[2] H. Esbensen et al., Phys. Rev. B 18, 1039 (1978).

[3] M. Clément et al., Nucl. Instrum. Methods Phys. Res. B 84, 434 (1995).

[4] S. Datz, H. F. Krause, C. R. Vane, H. Knudsen, P. Grafström, and R. H. Schuch, Phys. Rev. Lett. 77, 2995 (1996).

[5] J. Lindhard and A. H. Sørensen, Phys. Rev. A 53, 2443 (1996).
[6] U. Pfützner et al., Nucl. Instrum. Methods Phys. Res. B 86, 213 (1994).

[7] U. Fano, Annu. Rev. Nucl. Sci. 13, 67 (1963).

[8] H. Esbensen and J. A. Golovchenko, Nucl. Phys. A 298, 382 (1978).

[9] V. M. Biryukov, V. I. Kotov, and Yu. A. Chesnokov, Crystal Channeling and its Applications at High Energy Accelerators (Springer, Berlin, 1997). 